\title{
EXPLICIT FORMULA FOR WEIGHTED SCALAR NONLINEAR HYPERBOLIC CONSERVATION LAWS
}

\author{
PHILIPPE LE FLOCH AND JEAN-CLAUDE NEDELEC
}

ABSTRACT. We prove a uniqueness and existence theorem for the entropy weak solution of nonlinear hyperbolic conservation laws of the form

$$
\frac{\partial}{\partial t}(r u)+\frac{\partial}{\partial x}(r f(u))=0,
$$

with initial data and boundary condition. The scalar function $u=u(x, t)$, $x>0, t>0$, is the unknown, the function $f=f(u)$ is assumed to be strictly convex with inf $f(\cdot)=0$ and the weight function $r=r(x), x>0$, to be positive (for example, $r(x)=x^{\alpha}$, with an arbitrary real $\alpha$ ).

We give an explicit formula, which generalizes a result of P. D. Lax. In particular, a free boundary problem for the flux $r(\cdot) f(u(\cdot, \cdot))$ at the boundary is solved by introducing a variational inequality. The uniqueness result is obtained by extending a semigroup property due to B. L. Keyfitz.

0. Introduction. We consider weighted scalar nonlinear hyperbolic conservation laws of the form

$$
\frac{\partial}{\partial t}(r u)+\frac{\partial}{\partial x}(r f(u))=0,
$$

where the scalar function $u=u(x, t), x>0, t>0$, is the unknown. The flux function $f=f(u)$ is assumed to be strictly convex with inf $f(\cdot)=0$ and the weight function $r=r(x), x>0$, to be positive.

This paper is concerned with the mixed problem associated with the equation (0.1): we are looking for a solution $u=u(x, t)$ of $(0.1)$, satisfying an initial data and a boundary condition. But, it is well known that conservation laws of the type (0.1) do not possess classical solutions, even when the initial data is smooth: discontinuities appear in finite time. Hence, we consider only weak solutions of $(0.1)$, that is solutions in the sense of distributions. And, for the sake of uniqueness, we have to add an entropy condition that selects the physical (or entropy) solution among all the solutions in the sense of distributions. For a convex flux function $f(\cdot)$, the entropy condition is written as

$$
u(x-0, t) \geq u(x+0, t), \quad x>0, t>0 .
$$

(See P. D. Lax [3] and O. A. Oleinik [5].)

We are interested (in particular) in the case where the function $r=r(x)$ tends to zero at the point $x=0$ or at the point $x=+\infty$. For example, we can take $r(x)=x^{\alpha}(\alpha \in \mathbf{R})$. In such a case, the equation (0.1) possesses an algebraic

Received by the editors April 9, 1986 and, in revised form, September 9, 1986 and March 23, 1987.

1980 Mathematics Subject Classification (1985 Revision). Primary 36L65, 35D05, 35C05; Secondary $76 \mathrm{~N} 15,35 \mathrm{~L} 67$. 
singularity and generally a solution $u(\cdot, \cdot)$ is not bounded in the neighborhood of the origin $(x=0)$ or at infinity $(x=+\infty)$. These situations are interesting, because weighted conservation laws of the form (0.1) appear in fluid dynamics with spherical or cylindrical symmetry. (See G. B. Whitham [9]). The usual uniqueness and existence theorems $([\mathbf{2}-\mathbf{6}]$ and Smoller $[\mathbf{8}])$ are not valid in that case.

The main difficulties for a uniqueness and existence theory for the equation $(0.1)$ are to have a good formulation of the boundary condition (at the points $x=0$ or $+\infty)$ and to determine the functional spaces to which a solution of $(0.1)$ belongs. Namely, while we fix an initial condition as

$$
u(x, 0)=u_{0}(x), \quad x>0,
$$

with a given function $u_{0}(\cdot)$, we really cannot impose such a condition at the boundary. The boundary condition is necessarily linked to the entropy condition. We will establish that the functions $r(\cdot) f(u(\cdot, \cdot))$ and $\operatorname{sgn} f^{\prime}(u(\cdot, \cdot))$ possess traces at the boundary in a weak sense. And, we will follow ideas of Bardos-Leroux-Nedelec $[\mathbf{1}]$ : if $\gamma_{0}(\cdot)$ (respectively $\varepsilon_{0}(\cdot, \cdot)$ ) denotes the trace of $r(\cdot) f(u(\cdot, \cdot))$ (resp. $\operatorname{sgn} f^{\prime}(u(\cdot, \cdot))$ ) at $x=0$, for example, the boundary condition at $x=0$ is written as (see $\S 1$ )

$$
\left\{\begin{array}{lll}
\gamma_{0}(t)=W_{0}(t) \quad \text { and } \quad \varepsilon_{0}(t)=+1, \quad \text { or } \\
\gamma_{0}(t) \geq W_{0}(t) \quad \text { and } \quad \varepsilon_{0}(t)=-1, \quad t>0
\end{array}\right.
$$

with a given function $W_{0}(\cdot) \geq \inf f(\cdot)$ (we shall see that it is not restrictive).

To obtain an existence result concerning the entropy weak solution of the weighted conservation laws $(0.1)$ with initial data and boundary conditions, we extend the explicit representation derived by P. D. Lax [2] (for conservation laws of the form (0.1) with $r(\cdot) \equiv 1$ and without boundary data, when the variable $x$ describes $\mathbf{R}$ ). Furthermore, for the sake of uniqueness, we establish a $L^{1}$-semigroup property in the class of piecewise regular solutions, which generalizes a previous result of B. L. Keyfitz [6]. For example, if the weight function $r(\cdot)$ is constant, and if $u(\cdot, \cdot)$ and $v(\cdot, \cdot)$ are entropy weak solutions associated with (integrable) initial data $u_{0}(\cdot)$ and $v_{0}(\cdot)$ and (bounded) boundary data $\bar{u}_{0}(\cdot)$ and $\bar{v}_{0}(\cdot)$, we show that

$$
\begin{aligned}
\int_{0}^{+\infty}|u(x, t)-v(x, t)| d x \leq & \int_{0}^{+\infty}\left|u_{0}(x)-v_{0}(x)\right| d x \\
& +\int_{0}^{t} \mid f\left(\bar{u}_{0}(s)-f\left(\bar{v}_{0}(s) \mid d s, \quad t \geq 0,\right.\right.
\end{aligned}
$$

where we suppose that $f^{\prime}\left(\bar{u}_{0}(\cdot)\right)$ and $f^{\prime}\left(\bar{v}_{0}(\cdot)\right)$ are positive functions.

Note that we have to solve a free boundary problem: an explicit formula is derived for the flux $r(\cdot) f(u(\cdot, \cdot))$ at the boundary by using a variational inequality. This inequality determines, for example at point $x=0$, for $t>0$, if the value $u(0+, t)$ is incoming that is $\varepsilon_{0}(t)=+1$, or if it is outcoming that is $\varepsilon_{0}(t)=-1$ with previous notations (see $\S 1$ ).

An interesting model equation is the weighted Burgers equation $(\alpha \in \mathbf{R})$ :

$$
\frac{\partial}{\partial t}\left(x^{\alpha} u(x, t)\right)+\frac{\partial}{\partial x}\left(x^{\alpha} \frac{u(x, t)^{2}}{2}\right)=0, \quad x>0, t>0,
$$

for which a straightforward change of unknown function leads to the exact solution of this equation: $v(y, t)=(\alpha / 2+1) x^{\alpha} u(x, t), y=x^{\alpha / 2+1}$. For details, we refer the reader to $[\mathbf{1 0}]$. 
A different approach about the algebraic singularities in the equation (0.1), can be found in the recent paper of M. E. Schonbek [7], which uses the classical viscosity method and the theory of compensated compactness to obtain an existence result.

1. Formulation of the problem. Let $f=f(u)$ and $r=r(x)$ be such that

$$
f(\cdot) \in C^{2}(\mathbf{R}), \quad a^{\prime}(\cdot)>0, \quad \lim _{|u| \rightarrow \infty} \frac{f(u)}{|u|}=\infty, \quad f\left(u_{*}\right)=a\left(u_{*}\right)=0,
$$

with a real $u_{*}$, and

$$
r(\cdot) \in C^{1}\left(\mathbf{R}_{+} ; \mathbf{R}_{+}\right)
$$

with $a(\cdot)=f^{\prime}(\cdot)$. Let $b$ be the inverse function of $a: b=a^{-1}$. Take the initial data $u_{0}=u_{0}(x)$; and the boundary data $w_{0}=w_{0}(t)$ and $w_{\infty}=w_{\infty}(t)$, which are assumed to be nonnegative. Then, consider the following problem with the unknown $u=u(x, t) \in \mathbf{R}$, for $x$ and $t$ positive:

$$
\begin{aligned}
\frac{\partial}{\partial t}(r u)+\frac{\partial}{\partial x}(r f(u)) & =0, \\
u(x, 0)=u_{0}(x), & x>0,
\end{aligned}
$$

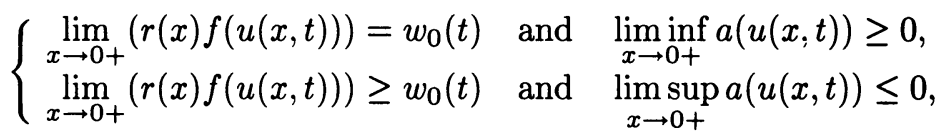

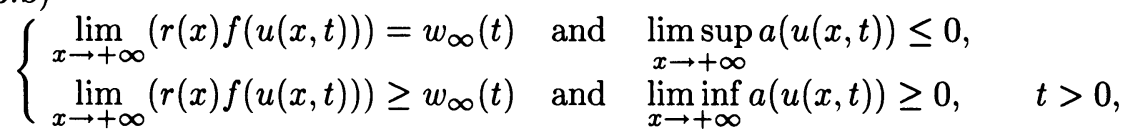

$$
u(x-0, t) \geq u(x+0, t), \quad x>0, t>0 .
$$

In this paper, a complete uniqueness and existence result is proved for the problem (1.1)-(1.2)-(1.4) with either the boundary condition (1.3.a), or (1.3.b) or with both (1.3.a) and (1.3.b).

In the second section, we study the characteristic curves associated with the weighted scalar nonlinear hyperbolic conservation law (1.1), according to the behaviour of the flux function $f(\cdot)$ at $u_{*}$ and at infinity, and to the one of the weight function $r(\cdot)$ at zero and at infinity. Note that this study will be essential to derive the explicit formula for a solution of (1.1)-(1.4) and to impose the boundary conditions at $x=0$ (see (1.3.a)) or at $x=+\infty$ (see (1.3.b)).

The main results are expressed in $\S \S 4-6$ : under hypotheses on the behaviour of the functions $f(\cdot)$ and $r(\cdot)$, that seem to be the "most physical" ones (see assumption (H.3)), the problem (1.1), (1.2), (1.4) with the boundary condition (1.3.a) (at $x=0$ ) is well posed. The uniqueness of the solution is deduced from a semigroup property in $L^{1}$-space. An explicit formula is derived for the solution; in particular, the flux $r(x) f(u(x, t))$ admits a limit when $x$ tends to zero and this limit satisfies a straightforward variational inequality. For example, the hypotheses of $\S \S 4-6$ are satisfied by the weighted Burgers equations (0.6) when $\alpha$ is greater than -2 .

Finally, in $\S 7$, we view all the other possible behaviours of the functions $f(\cdot)$ and $r(\cdot)$ : according to these behaviours, the problem $(1.1),(1.2),(1.4)$ is well posed 
whether only with the boundary condition (1.3.b) at $x=+\infty$ (see further the assumption (H.4)), or with no boundary condition (see further (H.3.b), (H.4.a)), or with both the boundary conditions (1.3.a) and (1.3.b) (see (H.3.a), (H.4.b)). In this last case, it will be assumed, to simplify, that the two boundary data $w_{0}(\cdot)$ and $w_{\infty}(\cdot)$ do not lead to two free boundary problems. The complete uniqueness and existence result is given, with the semigroup property and the explicit representation.

REMARK 1. (1) In view of previous results [1] concerning the equation (1.1), the boundary conditions (1.3.a) and (1.3.b) seem to be "natural". We consider that they are clearly justified by the semigroup property in weighted $L^{1}$-space, which will be established in $\S 6$.

(2) The hypothsis inf $f(\cdot)=0$ included in (H.1) is probably not essential, but, without this one, difficulties appear in the study of the characteristic curves.

2. Study of the characteristic curves. The study of the characteristic curves will be essential to derive the explicit formula. Thus, we distinguish here between the different behaviours of these ones, according to the behaviours of the flux function $f(\cdot)$ and the weight function $r(\cdot)$. More precisely, we define two reciprocal functions of the convex function $f(\cdot)$, denoted by $f_{+}^{-1}(\cdot)$ and $f_{-}^{-1}(\cdot)$, as follows:

$$
\begin{gathered}
f_{+}^{-1}:\left[0,+\infty\left[\rightarrow \left[u_{*} ;+\infty\left[, \quad v \mapsto u, \quad v=f(u) \quad \text { with } u \geq u_{*},\right.\right.\right.\right. \\
f_{-}^{-1}:\left[0,+\infty[\rightarrow]-\infty ; u_{*}\right], \quad v \mapsto u, \quad v=f(u) \quad \text { with } u \leq u_{*} .
\end{gathered}
$$

And, we consider the functions $h_{+}(\cdot, \cdot)$ and $h_{-}(\cdot, \cdot)$ given by

$$
h_{\varepsilon}(c, \xi)=\frac{1}{a\left[f_{\varepsilon}^{-1}(c / r(\xi))\right]}, \quad \xi>0, c>0, \varepsilon= \pm .
$$

The behaviour of the characteristic curves will depend on one of these functions $h_{+}(\cdot, \cdot)$ or $h_{-}(\cdot, \cdot)$ when the variable $\xi$ tends to zero or to infinity.

First, recall that a characteristic curve associated with the hyperbolic equation (1.1) is a (regular) curve $X=X(s)$ that verifies the differential equation

$$
\frac{d}{d s} X(\cdot)=a(u(X(\cdot), \cdot))
$$

where the function $u=u(x, t)$ is assumed to be a (regular) solution of (1.1). By using (1.1), it is easy to show that such a curve is a solution of

$$
\frac{d}{d s}\left\{r(X(\cdot)) f\left[b\left(\frac{d}{d s} X(\cdot)\right)\right]\right\}=0
$$

which is an O.D.E. depending only on the data $r(\cdot), f(\cdot)$ and $b(\cdot)$. So, in virtue of the definitions (2.1) and (2.2) of the functions $f_{+}^{-1}(\cdot)$ and $f_{-}^{-1}(\cdot)$, one deduces from $\left(2.4^{\prime}\right)$ the two following ordinary differential equations:

$$
\begin{aligned}
& \frac{d}{d s} X(\cdot)=a\left[f_{+}^{-1}\left(\frac{c}{r(X(\cdot))}\right)\right] \\
& \frac{d}{d s} X(\cdot)=a\left[f_{-}^{-1}\left(\frac{c}{r(X(\cdot))}\right)\right]
\end{aligned}
$$


with an arbitrary positive constant $c$. Here, we use the assumptions $\inf f(\cdot)=0$ and $r(\cdot)>0$.

For any $x$ and $t$ positive and for each positive constant $c$, in virtue of hypotheses (H.1) and (H.2), the classical Cauchy-Lipschitz theorem gives the existence of a curve $X=X(s)$ and a maximal interval $[\tau ; t]$ such that equation (2.5a) (respectively $(2.5 \mathrm{~b}))$ is verified on $[\tau ; t]$ and $X(t)=x$. If the point $\tau$ equals zero, we define the point $y$ as $y=X(0)$, and we take $y=0$ if the point $\tau$ is positive. Then, with the definition (2.3) of the functions $h_{+}$and $h_{-}$, we deduce by integration from (2.5a) or $(2.5 \mathrm{~b})$ the property

$$
t-\tau=\int_{y}^{x} h_{\varepsilon}(c, \xi) d \xi, \quad \varepsilon= \pm,
$$

since the point $(y, \tau)$ is the origin of the characteristic curve associated with the constants $c$ and $\varepsilon$.

To study the set described by the previous point $(y, \tau)$ when the two constants $c$ and $\varepsilon$ describe $[0 ;+\infty[$ and $\{-1,+1\}$ respectively, the following assumptions will be considered:

(H.3.a) $h_{+}(c, \cdot) \in L^{1}(0,1), \forall c>0$

(H.3.b) $h_{-}(c, \cdot) \notin L^{1}(1, \infty), \forall c>0$;

(H.4.a) $h_{+}(c, \cdot) \notin L^{1}(0,1), \forall c>0$

(H.4.b) $h_{-}(c, \cdot) \in L^{1}(1, \infty), \forall c>0$;

(H.5) $h_{-}(c, \cdot) \in L^{1}(0,1), \forall c>0$;

(H.6) $h_{-}(c, \cdot) \notin L^{1}(0,1), \forall c>0$;

(H.7) $h_{+}(c, \cdot) \in L^{1}(1, \infty), \forall c>0$;

(H.8) $h_{+}(c, \cdot) \notin L^{1}(1, \infty), \forall c>0$.

We note that hypothesis (H.3.b), for example, means that

$$
\lim _{x \rightarrow+\infty} \int_{1}^{x} h_{-}(c, \xi) d \xi=-\infty, \quad \forall c>0,
$$

because the functions $h_{\varepsilon}(\cdot, \cdot)$ possess a defined sign.

REMARK 2. (1) Since the flux function $f(\cdot)$ is convex, one can show that the opposites of the assumptions (H.3.a), (H.3.b), (H.5) and (H.7) are exactly (H.4.a), (H.4.b), (H.6) and (H.8) respectively.

(2) Hypotheses (H.3) and (H.4) (respectively (H.5)-(H.8)) are concerned with the characteristic curves that are incoming (resp. outcoming).

We begin with hypothesis (H.3), which seems to be the most "physical" one. (It is satisfied by the weighted Burgers equations with $\alpha>-2$.) Let

$$
E=\{(x, t ; y, \tau) \mid x, y \geq 0, t>\tau \geq 0, y \tau=0 \text { and }(x>0 \text { or } \tau=0)\},
$$

and define the function

$$
\varepsilon(x, t ; y, \tau)= \begin{cases}+1 & \text { if } y<x \\ -1 & \text { if } y \geq x\end{cases}
$$

Lemma 1. Assume that the hypotheses (H.1)--(H.3) are satisfied. Then, for each point $(x, t ; y, \tau)$ into the set $E$, there exists one and only one constant $c(x, t ; y, \tau)$ in $[0 ;+\infty]$ and one and only one curve $X=X(s)$ such that

$$
\left\{\begin{array}{l}
r\left(X(\cdot) f\left(b\left(X^{\prime}(\cdot)\right)\right)=c(x, t ; y, \tau),\right. \\
X(t)=x, \quad \text { if } \tau=0, \quad X(\tau)=0 \quad \text { if } y=0 .
\end{array}\right.
$$


The nonnegative function $c(\cdot ; \cdot, \cdot)$, defined over $E$, satisfies the following properties:

$$
\begin{gathered}
t-\tau=\int_{y}^{x} h_{\varepsilon(x, t ; y, \tau)}(c(x, t ; y, \tau), \xi) d \xi, \quad(x, t ; y, \tau) \in E, y \neq x, \\
c(x, t ; x, 0)=0, \quad x>0, t>0 .
\end{gathered}
$$

Moreover, we have the following facts:

$$
\begin{gathered}
\left\{\begin{array}{l}
\forall x>0, \forall t>0, \text { the function: }[x ;+\infty[\ni y \mapsto c(x, t ; y, 0) \\
\ni[0 ;+\infty[\text { is an increasing bijection. }
\end{array}\right. \\
\left\{\begin{array}{l}
\forall x>0, \forall t>0, \text { the function: }[0 ; x] \ni y \mapsto c(x, t ; y, 0) \\
\ni[0 ; c(x, t ; 0,0)] \text { is a decreasing bijection. }
\end{array}\right. \\
\left\{\begin{array}{l}
\forall x>_{\neq} 0, \forall t>0, \text { the function: }[0 ; t[\ni \tau \mapsto c(x, t ; 0, \tau) \\
\ni[c(x, t ; 0,0) ;+\infty] \text { is an increasing bijection. }
\end{array}\right.
\end{gathered}
$$

If Hypothesis (H.5) (respectively (H.6)) is satisfied, then the property (2.12) holds with $x=0$ (resp.:

$$
\left.\lim _{x \rightarrow 0+} c(x, t ; y, 0)=+\infty, \quad \forall t>0, \forall y>0\right) .
$$

If Hypothesis (H.7) (respectively (H.8)) is satisfied, then the property (2.13) holds with $x=+\infty$ (resp.:

$$
\left.\lim _{x \rightarrow+\infty} c(x, t ; y, 0)=+\infty, \quad \forall t>0, \forall y>0\right) .
$$

The proof of Lemma 1 is easy, so we omit it.

In order to examine Hypotheses (H.4)-(H.8), the previous result will be extended as follows:

LEMMA 2. Under Hypotheses (H.1) and (H.2), there exists one and only one function $c=c(x, t ; y, \tau) \in[0 ;+\infty]$ that verifies the property

$$
t-\tau=\int_{y}^{x} h_{\varepsilon(x, t ; y, \tau)}(c(x, t ; y, \tau), \xi) d \xi
$$

for any point $(x, t ; y, \tau)$ such that

$$
0 \leq x \leq+\infty, \quad 0 \leq y \leq+\infty, \quad 0 \leq \tau<t \quad(y \tau=0 \text { or } \tau / y=0) .
$$

Moreover, for any positive numbers $x$ and $t$, we have the following facts:

(1) If (H.3.a) holds, the function $c(x, t ; \cdot, \cdot)$ defined from $\{(y, 0) \mid 0 \leq y<x\} \vee$ $\{(0, \tau) \mid 0<\tau<t\}$ into $] 0 ;+\infty[$ is a decreasing bijection.

(2) If (H.4.a) holds, the function $c(x, t ; \cdot, \cdot)$ defined from $\{(y, 0) \mid 0<y<x\}$ into ] $0 ;+\infty[$, is a decreasing bijection.

(3) If (H.3.b) holds, the function $c(x, t ; \cdot, \cdot)$ defined from $\{(y, 0) \mid x<y<+\infty\}$ into $] 0 ;+\infty]$, is an increasing bijection.

(4) If (H.4.b) holds, the function $c(x, t ; \cdot, \cdot)$ defined from $\{(y, 0) \mid x<y \leq+\infty\} \vee$ $\{(+\infty, \tau) \mid 0<\tau<t\}$ into $] 0 ;+\infty[$ is an increasing bijection.

Furthermore, when the variable $x$ tends to zero or infinity, with $t, y, \tau$ fixed, the value $c(x, t ; y, \tau)$ tends to infinity, except in the following cases:

(5) If (H.5) holds, then properties (3) and (4) remain valid with $x$ equal to zero.

(6) If (H.7) holds, then properties (1) and (5) remain valid with $x$ equal to infinity.

REMARK 3 . Here, the function $\varepsilon=\varepsilon(x, t ; y, \tau)$ given by $(2.8)$ has been extended clearly to any point $(x, t ; y, \tau)$ that verifies $(2.18)$. 
3. Main results. In order to apply Lemma 1, (H.1)-(H.3) are now considered and we give the complete uniqueness and existence result concerning problem (1.1), (1.2), (1.4) with boundary condition (1.3.a).

THEOREM 1 (EXISTENCE). Under (H.1)-(H.3) and if the function $r(\cdot) u_{0}(\cdot)$ is integrable and the functions $r(\cdot) f\left(u_{0}(\cdot)\right)$ and $w_{0}(\cdot)$ are measurable and bounded, there exists a solution of problem (1.1), (1.2), (1.3.a), (1.4), $u=u(x, t)$, which possesses the following regularity: the function $u(\cdot, \cdot)$ is piecewise continuous having limits on left and on right at each point with respect to the variables $x>0$ and $t>0$; the function $\operatorname{sgn} a(u(\cdot, \cdot))$ admits a measurable trace at the point $x=0$; and, the function $r(\cdot) f(u(\cdot, \cdot))$ admits at the point $x=0$ a bounded measurable trace.

Moreover, the function $r(\cdot) f(u(\cdot))$ is bounded as follows:

$$
\|r(\cdot) f(u(\cdot, \cdot))\|_{\infty} \leq \sup \left\{\left\|r(\cdot) f\left(u_{0}(\cdot)\right)\right\|_{\infty},\left\|w_{0}(\cdot)\right\|_{\infty}\right\}
$$

With suitable regularity about the data $u_{0}(\cdot)$ and $w(\cdot)$, one can show that the solution $u(\cdot, \cdot)$ given by Theorem 1 is more regular. For example, if the data are piecewise continuously differentiable, then the solution possesses the same structure, at the only eventual exception of some curves.

Without additional assumptions concerning the data, we will see that the traces at $t=0$ and at $x=0$ in Theorem 1 hold in the following weak sense: there exist two sets $\mathcal{E}$ and $\mathcal{f}$ of zero measure, included in the interval $[0,1]$, such that

$$
\lim _{\substack{t \rightarrow 0+\\ t \notin \mathcal{E}}} \int_{0}^{x} u(\xi, t) r(\xi) d \xi=\int_{0}^{x} u_{0}(\xi) r(\xi) d \xi, \quad x \geq 0
$$

and

$$
\lim _{\substack{x \rightarrow 0+\\ x \notin \mathcal{F}}} \int_{0}^{t} f(u(x, s)) r(x) d s=\int_{0}^{t} \gamma(s) d s, \quad t \geq 0
$$

where the trace of $r(\cdot) f(u(\cdot, \cdot))$ at $x=0$ will be denoted by $\gamma(\cdot)$.

The uniqueness is specified by a semigroup property in weighted $L^{1}$-space as follows:

THEOREM 2 (UNIQUENESS). Under (H.1)-(H.3), let $u(\cdot, \cdot)$ and $v(\cdot, \cdot)$ be piecewise continuously differentiable solutions of problem (1.1), (1.2), (1.3.a), (1.4), associated with initial data $u_{0}(\cdot)$ and $v_{0}(\cdot)$ and (nonnegative) boundary data $w_{0}(\cdot)$ and $z_{0}(\cdot)$ respectively. Then, if the functions $r(\cdot) u_{0}(\cdot)$ and $r(\cdot) v_{0}(\cdot)$ (respectively $w_{0}(\cdot)$ and $\left.z_{0}(\cdot)\right)$ are integrable (resp. locally integrable), we have for all $0 \leq t_{1} \leq t_{2}$ :

$$
\int_{0}^{+\infty}\left|(u-v)\left(x, t_{2}\right)\right| r(x) d x \leq \int_{0}^{+\infty}\left|(u-v)\left(x, t_{1}\right)\right| r(x) d x+\int_{t_{1}}^{t_{2}}\left|w_{0}(s)-z_{0}(s)\right| d s
$$

It results from Theorem 2 that if the initial data $u_{0}(\cdot)$ satisfies $r(\cdot) u_{0}(\cdot) \in$ $L^{1}(0, \infty)$ then the solution $u(\cdot, \cdot)$ satisfies $r(\cdot) u(\cdot, \cdot) \in L^{\infty}\left(\mathbf{R}_{t}^{+} ; L^{1}\left(\mathbf{R}_{x}^{+}\right)\right)$with

$$
\int_{0}^{+\infty}|u(x, t)| r(x) d x \leq \int_{0}^{+\infty}\left|u_{0}(x)\right| r(x) d x+\int_{0}^{t}\left|w_{0}(s)\right| d s, \quad t \geq 0 .
$$


Finally, define the function

$$
\begin{aligned}
G(x, t ; y, \tau)= & \int_{0}^{y} u_{0}(\xi) r(\xi) d \xi-\int_{0}^{\tau} \gamma(s) d s-(t-\tau) c(x, t ; y, \tau) \\
& +\int_{y}^{x} f_{\varepsilon(x, t ; y, \tau)}^{-1}\left(\frac{c(x, t ; y, \tau)}{r(\xi)}\right) r(\xi) d \xi, \quad \forall(x, t ; y, \tau) \in E
\end{aligned}
$$

with an arbitrary (bounded measurable) function $\gamma=\gamma(s)$ to specify. Here, the set $E$ and the functions $\varepsilon$ and $c$ are given by (2.7), (2.8) and (2.10) respectively.

Then, the solution given by Theorem 1 admits the following explicit representation.

THEOREM 3 (EXPLICIT FORMULA). A. Characterization of the boundary flux $\gamma(\cdot)$. If (H.6) is satisfied, we have $\gamma(t)=w_{0}(t), t>0$.

If (H.5) is satisfied, we proceed as follows:

(A1) For any positive number $t$, let $y(t)$ be a point which minimizes the function: $[0 ;+\infty[\ni y \rightarrow G(0, t ; y, 0)$.

(A2) Let $m=m(t), t>0$, be the unique continuous nonnegative function, almost everywhere differentiable, given by

$$
\begin{aligned}
& m(0+)=0+, \\
& \left\{\frac{d}{d t} m(t)-w(t)+c(0, t ; y(t), 0)\right\} \times\{\varphi-m(t)\} \\
& \quad \forall \varphi \geq 0 \text { a.e. } t>0 . \\
& \forall \varphi \geq
\end{aligned}
$$

(A3) Define the function

$$
\gamma(t)=\frac{d}{d t} m(t)+c(0, t ; y(t), 0), \quad t>0 .
$$

B. Explicit representation inside. For any positive numbers $x$ and $t$, the value $u(x, t)$ is given by the formula

$$
u(x, t)=f_{\varepsilon(x, t ; y, \tau)}^{-1}\left(\frac{c(x, t ; y, \tau)}{r(x)}\right)
$$

where the point $(y, \tau)$ realizes the minimum value of the function $G(x, t ; \cdot, \cdot)$, when, in definition (3.5) the function $\gamma(\cdot)$ is given by (3.7).

Hypotheses (H.3) and (H.5) are satisfied if the function $r(\cdot)$ is assumed to be bounded in a neighborhood of the origin $(x=0)$ and to be minorized by a positive constant in a neighborhood of infinity $(x=+\infty)$. For example, one can take $r(x)=x^{\alpha}$, with an arbitrary nonnegative real $\alpha$.

We divide the proof of Theorems 13 into three steps. In $\S \S 4$ and 5 , we prove the existence of a solution with the explicit representation (Theorems 1 and 2 ). In $\S 6$ the proof of Theorem 2 is given.

4. First step: Explicit formula. Let $u=u(x, t)$ be a (sufficiently regular) solution of $(1.1),(1.2),(1.3 . \mathrm{a}),(1.4)$ and consider the function $\mathcal{U}(\cdot, \cdot)$ defined by

$$
\mathcal{U}(x, t)=\int_{0}^{x} u(\xi, t) r(\xi) d \xi, \quad x \geq 0, t \geq 0 .
$$


We take

$$
\gamma(t)=\lim _{x \rightarrow 0+}\{r(x) f(u(x, t))\}, \quad t \geq 0 .
$$

Integrating equation (1.1) yields

$$
\mathcal{U}_{t}(\xi, s)+r(\xi) f\left(\mathcal{U}_{x}(\xi, s)\right)-\gamma(s)=0, \quad \xi \geq 0, s \geq 0 .
$$

The hypothesis of convexity of $f(\cdot)$ gives

$$
f(u(\xi, s)) \geq f(v)+a(v)(u(\xi, s)-v), \quad \forall v \in \mathbf{R}, \forall \xi, s>0 .
$$

So, using (4.1) and (4.2), we obtain the following inequality:

$$
\mathcal{U}_{t}(\xi, s)+a(v) \mathcal{U}_{x}(\xi, s) \leq \gamma(s)+r(\xi)(a(v) v-f(v))
$$

for arbitrary $\xi \geq 0, s \geq 0$ and $v \in \mathbf{R}$.

Let $(x, t)$ be fixed and $X=X(s)$ be a characteristic curve such that $X(t)=x$. We are going to integrate the inequality (4.3) along the curve $X(\cdot)$, with $v=b\left(X^{\prime}(s)\right)$; we distinguish between two cases:

(1) $X(\cdot)$ is defined on the interval $[0 ; t]$. The point $X(0)$ is denoted by $y$; from (2.9) and (2.10) we deduce that

$$
\mathcal{U}(x, t)-\mathcal{U}(y, 0) \leq \int_{0}^{t} \gamma(s) d s+\int_{y}^{x} f_{\varepsilon}^{-1}\left(\frac{c}{r(\xi)}\right) r(\xi) d \xi-c t
$$

with $\varepsilon=\varepsilon(x, t ; y, 0)$ and $c=c(x, t ; y, 0)$. Thus, we obtain the inequality

$$
\mathcal{U}(x, t)-\int_{0}^{t} \gamma(s) d s \leq \int_{0}^{y} u_{0}(\xi) r(\xi) d \xi-c t+\int_{y}^{x} f_{\varepsilon}^{-1}\left(\frac{c}{r(\xi)}\right) r(\xi) d \xi
$$

By Lemma 1, (4.4) is valid for any nonnegative number $y$.

(2) $X(\cdot)$ is defined on an interval $[\tau ; t]$, where the point $\tau$ is such that $X(\tau)=0$. Here, we have

$$
\begin{aligned}
\int_{\tau}^{t} r & (X(s))\left[b\left(X^{\prime}(s)\right) X^{\prime}(s)-f\left(b\left(X^{\prime}(s)\right)\right)\right] d s \\
& =\int_{\tau}^{t} r(X(s)) f_{\varepsilon}^{-1}\left(\frac{c}{r(X(s))}\right) X^{\prime}(s) d s-\int_{\tau}^{t} r(X(s)) f\left(b\left(X^{\prime}(s)\right)\right) d s \\
& =-c(t-\tau)+\int_{0}^{x} f_{+}^{-1}\left(\frac{c}{r(\xi)}\right) r(\xi) d \xi .
\end{aligned}
$$

We obtain the inequality

$$
\mathcal{U}(x, t)-\int_{0}^{t} \gamma(s) d s \leq-\int_{0}^{\tau} \gamma(s) d s-c(t-\tau)+\int_{0}^{x} f_{+}^{-1}\left(\frac{c}{r(\xi)}\right) r(\xi) d \xi
$$

with $\varepsilon=\varepsilon(x, t ; 0, \tau)$ and $c=c(x, t ; 0, \tau)$. By Lemma $1,(4.5)$ is valid for any number $y$ belonging to $[0 ; t]$.

Using (4.4), (4.5) and (3.5), we obtain the inequality

$$
\mathcal{U}(x, t)-\int_{0}^{t} \gamma(s) d s \leq G(x, t ; y, \tau)
$$

for any point $(x, t ; y, \tau) \in E$. 
Equality holds in (4.6) if and only if the curve $X(\cdot)$, whose origin is the point $(y, \tau)$, satisfies the property $u(X(s), s)=b\left(X^{\prime}(s)\right)$. Using (2.9), we obtain (3.8), and formula $\mathrm{B}$ of Theorem 3 is proved.

When (H.5) is satisfied, we can consider the functions $c(0, \cdot ; \cdot, 0)$ and $G(0, t ; y, 0)$, and define the function $m=m(t)$ by

$$
m(t)=\inf _{y \geq 0}\left\{\int_{0}^{t} \gamma(s) d s+G(0, t ; y, 0)\right\} .
$$

And, for any positive number $t$, let $y(t)$ be a point which realizes the infimum in (4.7). We can prove that $m(t)$ and $y(t)$ exist and the function $y(\cdot)$ is nondecreasing. Moreover, the derivative of the function $m(\cdot)$ is (for proofs, see [10]):

$$
\frac{d}{d t} m(t)=\gamma(t)-c(0, t ; y(t), 0) .
$$

Formula A of Theorem 3 follows from these arguments.

Now when (H.5) is satisfied, to prove that $\gamma(t)=w_{0}(t), t>0$, it is sufficient to show that ( $\operatorname{see}(1.3 . \mathrm{a}))+\infty \geq \liminf _{x \rightarrow 0+} a(u(x, t)) \geq 0$. But assume that the value $u(0+, t)$ is "outcoming". Then because of the regularity of the solution $u(\cdot, \cdot)$, there exists a characteristic curve $X(\cdot)$ defined on $[\tau ; t]$, along which $r(x) f(u(x, t))$ is constant. But if $c$ is such that

$$
r(X) f\left(b\left(X^{\prime}(t)\right)\right)=c, \quad X(t)=0,
$$

then

$$
\int_{0}^{X(t)} \frac{d \xi}{a\left[f_{-}^{-1}(c / r(\xi))\right]}=-(t-\tau)
$$

which is impossible, because $h_{-}(c, \cdot) \nmid \ni L^{1}(0,1)$.

5. Second step: Existence of a solution. We want to define a function $u=u(x, t)$ by formulas A and B of Theorem 3. For this purpose, as in Lax [4], we have:

LEMMA 3. Formulas A and B define the unique functions $y=y(t), m=m(t)$, $\gamma=\gamma(t)$ (if (H.5) is satisfied), $(y, \tau)=(y(x, t), \tau(x, t))$ and $u=u(x, t)$. The function $m(\cdot)$ is continuous and a.e. differentiable. The functions $y(\cdot), y(\cdot, \cdot), \tau(\cdot, \cdot)$ and $u(\cdot, \cdot)$ are piecewise continuous, and the functions $y(\cdot), y(x, \cdot), y(\cdot, t),-\tau(x, \cdot)$ and $-\tau(\cdot, t)$ are nondecreasing.

These monotonicity properties will be very useful to prove the boundary condition (1.3.a) and the entropy condition (1.4).

PROOF OF LEMMA 3. It suffices to prove that the functions $y(\cdot), y(\cdot, \cdot)$ and $\tau(\cdot, \cdot)$ are nondecreasing. For example, we will study the functions $y(\cdot, t)$ and $-\tau(\cdot, t)$.

(1) Let $t$ be positive and $x_{1}, x_{2}$ be such that $0<x_{1}<x_{2}$. To prove $y\left(x_{1}, t\right) \leq$ $y\left(x_{2}, t\right)$, it is sufficient to show that (we take $y_{1}=y\left(x_{1}, t\right)$ ) for all points $y$ belonging to $] 0 ; y_{1}[$, we have

$$
G\left(x_{2}, y_{1}\right)+G\left(x_{1}, y\right)<G\left(x_{2}, y\right)+G\left(x_{1}, y_{1}\right)
$$

(See the proof in [10]; we omit the variables $t$ and $\tau$ to simplify). Now, using (3.6), the inequality (5.1) is equivalent to

$$
H\left(x_{1}\right)<H\left(x_{2}\right)
$$


where the function $H(\cdot)$ is given by

$$
\begin{aligned}
H(x)= & t(c(x, y)-c(x, y))+\int_{y}^{x} f_{\varepsilon(x, y)}^{-1}\left(\frac{c(x, y)}{r(\xi)}\right) r(\xi) d \xi \\
& -\int_{y_{1}}^{x} f_{\varepsilon\left(x, y_{1}\right)}^{-1}\left(\frac{c\left(x, y_{1}\right)}{r(\xi)}\right) r(\xi) d \xi, \quad x>0 .
\end{aligned}
$$

But, by derivation, we obtain

$$
H^{\prime}(x)=r(x)\left(f_{\varepsilon(x, y)}^{-1}\left(\frac{c(x, y)}{r(x)}\right)-f_{\varepsilon\left(x, y_{1}\right)}^{-1}\left(\frac{c\left(x, y_{1}\right)}{r(x)}\right)\right) .
$$

The function $H^{\prime}(x)$ is positive because of the monotonicity of $c(x, \cdot)$ (Lemma 1), namely, if $x<y<y_{1}$, then $\varepsilon(x, y)=\varepsilon\left(x, y_{1}\right)=-1$. The functions $f_{-}^{-1}(\cdot)$ and $c(x, \cdot)$ are respectively decreasing and increasing. If $y<x<y_{1}$, then $\varepsilon(x, y)=$ $-\varepsilon\left(x, y_{1}\right)=+1$. The functions $f_{\varepsilon(x, y)}^{-1}(\cdot)$ and $f_{\varepsilon\left(x, y_{1}\right)}^{-1}(\cdot)$ are respectively decreasing and increasing. If $y<y_{1}<x$, then $\varepsilon(x, y)=\varepsilon\left(x, y_{1}\right)=+1$. The functions $f_{+}^{-1}(\cdot)$ and $c(x, \cdot)$ are respectively decreasing and increasing.

Finally, inequality (5.2) is verified and also (5.1). Thus, the function $y(\cdot, t)$ increases.

(2) Now, let $t$ be positive and $x_{1}, x_{2}$ such that $0<x_{1}<x_{2}$. To prove $\tau(x, t) \geq$ $\tau\left(x_{2}, t\right)$, it is sufficient to show that for all $\tau$ belonging to $] \tau_{1} ; t$, the following inequality holds:

$$
G\left(x_{2}, \tau_{1}\right)+G\left(x_{1}, \tau\right)<G\left(x_{2}, \tau\right)+G\left(x_{2}, \tau_{1}\right)
$$

where we take $\tau_{1}=\tau\left(x_{1}, t\right)$. Using (3.5) inequality (5.3) is rewritten as

$$
F\left(x_{1}\right)<F\left(x_{2}\right)
$$

with

$$
\begin{aligned}
F(x)= & -c(x, \tau)(t-\tau)+\int_{0}^{x} f_{+}^{-1}\left(\frac{c(x, \tau)}{r(\xi)}\right) r(\xi) d \xi-c\left(x, \tau_{1}\right)\left(t-\tau_{1}\right) \\
& +\int_{0}^{x} f_{+}^{-1}\left(\frac{c\left(x_{2}, \tau\right)}{r(\xi)}\right) r(\xi) d \xi, \quad x>0 .
\end{aligned}
$$

By derivation, we obtain

$$
F^{\prime}(x)=r(x)\left(f_{+}^{-1}\left(\frac{c(x, \tau)}{r(x)}\right)-f_{+}^{-1}\left(\frac{c\left(x, \tau_{1}\right)}{r(x)}\right)\right) .
$$

As previously, the monotonicity of $c(x, \cdot)$ insures that $F^{\prime}(\cdot)$ is positive. So, inequality (5.4) holds and $\tau(\cdot, t)$ decreases.

We now have to show that $u=u(x, t)$ satisfies properties (1.1), (1.2), (1.3.a) and (1.4). First, (1.1) will be shown. Define the set

$$
C_{t}=\{(y, \tau) \mid 0 \leq y, 0 \leq \tau<t, y \tau=0\}, \quad t>0,
$$

and the functions

$$
\begin{aligned}
& \Omega_{N}(x, t)=\int_{C_{t}} \exp (-N G(x, t ; \cdot, \cdot)) d \sigma \\
& u_{N}(x, t)=\frac{1}{\Omega_{N}(x, t)} \int_{C_{t}} f_{\varepsilon(x, t ; \cdot, \cdot)}^{-1}\left(\frac{c(x, t ; \cdot, \cdot)}{r(x)}\right) \exp (-N G(x, t ; \cdot, \cdot)) d \sigma
\end{aligned}
$$


and

$$
f_{N}(x, t)=\frac{1}{\Omega_{N}(x, t)} \int_{C_{t}} \frac{c(x, t ; \cdot, \cdot)}{r(x)} \exp (-N G(x, t ; \cdot, \cdot)) d \sigma,
$$

for $x>0, t>0$ and $N>0$. In view of formula $\mathrm{B}$, we have in the sense of distributions the convergences

$$
u_{N} \rightarrow u \text { and } f_{N} \rightarrow f(u) .
$$

Moreover, by taking $V_{N}(x, t)=\log \Omega_{N}(x, t)$, we have in the sense of distributions (we omit the variable $(x, t)$ )

$$
\frac{\partial V_{N}}{\partial t}=\frac{1}{\Omega_{N}} \int_{C_{t}}-N \frac{\partial G}{\partial t} \exp (-N G) d \sigma
$$

where, in virtue of (2.10) the time derivative $\partial G / \partial t$ is equal to

$$
\frac{\partial G}{\partial t}=-c-(t-\tau) \frac{\partial c}{\partial t}+\int_{y}^{x} h_{\varepsilon}(c, \xi) d \xi \frac{\partial c}{\partial t}=-c .
$$

We also obtain the space derivative of $V_{N}(\cdot, \cdot)$ as

$$
\frac{\partial V_{N}}{\partial x}=\frac{1}{\Omega_{N}} \int_{C_{t}}-N \frac{\partial G}{\partial x} \exp (-N G) d \sigma
$$

where, in virtue of $(2.10)$ we have

$$
\frac{\partial G}{\partial x}=-(t-\tau) \frac{\partial c}{\partial x}+f_{\varepsilon}^{-1}\left(\frac{c}{r}\right) r+(t-\tau) \frac{\partial c}{\partial x}=r f_{\varepsilon}^{-1}\left(\frac{c}{r}\right) .
$$

Thus, the derivatives of the function $V_{N}(\cdot, \cdot)$ are

$$
\frac{\partial V_{N}}{\partial t}=+N r f_{N} \quad \text { and } \quad \frac{\partial V_{N}}{\partial x}=-N r u_{N} .
$$

So, the two functions $u_{N}(\cdot, \cdot)$ and $f_{N}(\cdot, \cdot)$ are such that

$$
\frac{\partial}{\partial t}\left(r u_{N}\right)+\frac{\partial}{\partial x}\left(r f_{N}\right)=0 .
$$

Using (5.5) when $N$ tends to infinity, we deduce that the function $u(\cdot, \cdot)$ satisfies the weighted conservation law (1.1).

Now without new difficulty, as in [3], we can show properties (3.2) and (3.3). For details, see [10].

For property (1.3.a), we remark that the monotonicity of the functions $y(\cdot, \cdot)$ and $\tau(\cdot, \cdot)$, implies that the function $a(u(x, t))$ admits a sign for $x=0$ and $t>0$ : If $\lim _{x \rightarrow 0+} y(x, t) \geq 0$, then

$$
\liminf _{x \rightarrow 0+} a(u(x, t))=\lim _{x \rightarrow 0+} a(u(x, t)) \in[0 ;+\infty] .
$$

If $\lim _{x \rightarrow 0+} \tau(x, t) \leq 0$, then

$$
\limsup _{x \rightarrow 0+} a(u(x, t))=\lim _{x \rightarrow 0+} a(u(x, t)) \in[-\infty ; 0] .
$$

When (H.6) is satisfied, $\gamma(\cdot)$ is equal to the boundary data $w_{0}(\cdot)$; hence, the boundary condition (1.3.a) is clearly satisfied. When (H.6) is satisfied, we have that, for almost every $t>0$, if $m(t)>0$, then with the variational inequality, (3.6) gives

$$
\frac{d}{d t} m(t)=w(t)-c(0, t ; y(t), 0) .
$$


Hence by (3.7) $\gamma(t)=w(t)$ and the boundary condition (1.3.a) is satisfied.

If $m(t)=0$, and $d m(t) / d t=0$ (recall that $m$ is continuous)

$$
\gamma(t)=c(0, t ; y(t), 0)
$$

and simple arguments $[\mathbf{1 0}]$ lead to $\lim _{x \rightarrow 0+} a(u(x, t)) \in[-\infty ; 0]$. Hence, property (1.3.a) is satisfied.

Using the monotonicity of the functions $c(x, t ; \cdot, \cdot)$ (see Lemma 1) and $y(\cdot, t)$ and $\tau(\cdot, t)$ (see Lemma 3 ), we now prove the inequality

$$
f_{\varepsilon_{-}}^{-1}\left(c_{-} / r(x)\right) \geq f_{\varepsilon_{+}}^{-1}\left(c_{+} / r(x)\right),
$$

for any positive numbers $x$ and $t$, where we take

$$
\varepsilon_{ \pm}=\varepsilon(x, t ; y(x \pm 0, t), \tau(x \pm 0, t)), \quad c_{ \pm}=c(x, t ; y(x \pm 0, t), \tau(x \pm 0, t)) .
$$

Then, the entropy condition (1.4) will be deduced from (5.7) just by multiplying by the positive number $r(x)$. Now, we distinguish between several cases:

(1) If $\varepsilon_{+}=\varepsilon_{-}=-1$, then by property (2.12) the function $y \mapsto c(x, t ; y, 0)$ is increasing and by Lemma $3, x \mapsto y(x, t)$ is nondecreasing; but, the function $f_{-}^{-1}(\cdot)$ decreases.

(2) If $\varepsilon_{-}=+1$ and $\varepsilon_{+}=-1$, then (5.7) is obvious since $f_{+1}^{-1}(\cdot) \geq 0 \geq f_{-}^{-1}(\cdot)$.

(3) If $\varepsilon_{-}=\varepsilon_{+}=+1$ and $y(x-0, t)>0$, then by (2.13) the function $y \mapsto$ $c(x, t ; y, 0)$ is decreasing; moreover, $x \rightarrow y(x, t)$ is nondecreasing and the function $f_{+}^{-1}(\cdot)$ increases.

(4) If $\varepsilon_{-}^{-}=\varepsilon_{+}=+1, \tau(x-0, t)>0$ and $y(x+0, t)<0$, then by (2.13) and (2.14) we have $c_{-} \geq c(x, t ; 0,0) \geq c_{+}$.

(5) If $\varepsilon_{-}=\varepsilon_{+}=-1$ and $\tau(x+0, t)>0$, then by (2.14) the function $\tau \mapsto$ $c(x, t ; 0, \tau)$ is increasing; moreover, $x \rightarrow \tau(x, t)$ is nonincreasing and $f_{+}^{-1}(\cdot)$ increases.

Thus, in any case, inequality (5.7) results from these arguments. Inequality (5.7) shows that the two characteristic curves associated with the points $(y(x-0), \tau(x-0))$ and $(y(x+0), \tau(x+0))$ do not intersect.

Finally, it remains to show that the solution $u(\cdot, \cdot)$ satisfies the estimation (3.1). It is sufficient to prove that, for $x \geq 0$ and $t>0$, if a point $(y, \tau)$ satisfies the inequality

$$
c(x, t ; y, \tau) \geq \sup \left(\left\|r(\cdot) f\left(u_{0}(\cdot)\right)\right\|_{\infty},\|w(\cdot)\|_{\infty}\right)
$$

then for all points $\left(y^{\prime}, \tau^{\prime}\right)$ such that

$$
c^{\prime}=c\left(x, t ; y^{\prime}, \tau^{\prime}\right)>_{\neq} c=c(x, t ; y, \tau),
$$

the following property holds:

$$
G\left(x, t ; y^{\prime}, \tau^{\prime}\right)>_{\neq} G(x, t ; y, \tau)
$$


To fix the ideas, make the assumption that $x \leq y \leq y^{\prime}$ (the other cases are similar). Then, we show inequality (5.10) as follows:

$$
\begin{aligned}
G\left(x, t ; y^{\prime}, \tau^{\prime}\right)-G(x, t ; y, \tau) & \int_{y}^{y^{\prime}} u_{0}(\xi) r(\xi) d \xi-t c^{\prime}+t c \\
= & \int_{y}^{x} f_{-}^{-1}\left(\frac{c^{\prime}}{r(\xi)}\right) r(\xi) d \xi-\int_{y}^{x} f_{-}^{-1}\left(\frac{c}{r(\xi)}\right) r(\xi) d \xi \\
> & -\int_{y}^{y^{\prime}}\left|f_{-}^{-1}\left(\frac{\left\|r(\cdot) f\left(u_{0}(\cdot)\right)\right\|_{\infty}}{r(\xi)}\right)\right| r(\xi) d \xi-\int_{y}^{y^{\prime}} f_{-}^{-1}\left(\frac{c}{r(\xi)}\right) r(\xi) d \xi \\
= & -\int_{y}^{y^{\prime}}\left\{f_{-}^{-1}\left(\frac{c}{r(\xi)}\right)+\left|f_{-}^{-1}\left(\frac{\left\|r(\cdot) f\left(u_{0}(\cdot)\right)\right\|_{\infty}}{r(\xi)}\right)\right|\right\} r(\xi) d \xi,
\end{aligned}
$$

and the last term is nonnegative by assumption (5.8).

6. Third step: Uniqueness of the solution. Here, we prove Theorem 2 by following the same ideas as B. L. Keyfitz [6]. Let $u(\cdot, \cdot)$ and $v(\cdot, \cdot)$ be two piecewise continuously differentiable solutions of (1.1), (1.2), (1.3.a) and (1.4) associated with initial data $u_{0}(\cdot)$ and $v_{0}(\cdot)$ and boundary data $w_{0}(\cdot)$ and $z_{0}(\cdot)$ respectively. Let us take curves $y_{p}=y_{p}(t), p \in \mathbf{Z}$, such that $y_{p}(t) \leq y_{p+1}(t), \operatorname{sgn}(u(x, t)-v(x, t))=$ $(-1)^{p}, \forall x \in\left[y_{p}(t) ; y_{p+1}(t)\right], \forall t \geq 0$. Then we view as in [6] that the curves $y_{p}(\cdot)$ are characteristic curves (which have been studied in §2) or shock curves for $u(\cdot, \cdot)$ or $v(\cdot, \cdot)$, that is curves along which the functions $u(\cdot, \cdot)$ or $v(\cdot, \cdot)$ are discontinuous.

Define the function

$$
I(t)=\int_{0}^{+\infty}|u(x, t)-v(x, t)| d x, \quad t \geq 0 .
$$

We may write

$$
I(t)=\sum_{p \in \mathbf{Z}}(-1)^{p} \int_{y_{p}(t)}^{y_{p+1}(t)}|u(x, t)-v(x, t)| r(x) d x .
$$

By derivation of (6.1) we obtain with (1.1) by taking $y_{p}=y_{p}(t)$ and $y_{p}^{\prime}=y_{p}^{\prime}(t)$ :

$$
\begin{aligned}
& \frac{d}{d t} I(t)=\sum_{p \in \mathbf{Z}}(-1)^{p}[r(x)(f(u(x, t))-f(v(x, t)))]_{y_{p}}^{y_{p+1}} \\
& -\sum_{p \in \mathbf{Z}}(-1)^{p}\left\{y_{p+1}^{\prime}\left(u\left(y_{p+1}, t\right)-v\left(y_{p+1}, t\right)\right) r\left(y_{p+1}\right)\right. \\
& \left.\quad-y_{p}^{\prime}\left(u\left(y_{p}, t\right)-v\left(y_{p}, t\right)\right) r\left(y_{p}\right)\right\} .
\end{aligned}
$$

And thus, we have

$$
\left\{\begin{aligned}
& \frac{d}{d t} I(t)=\sum_{p \in \mathbf{Z}}(-1)^{p}\left\{r\left(y_{p+1}\right)(\right.\left.(f(u)-f(v))\left(y_{p+1}, t\right)-y_{p+1}^{\prime}(u-v)\left(y_{p+1}, t\right)\right) \\
&\left.-r\left(y_{p}\right)\left((f(u)-f(v))\left(y_{p}, t\right)-y_{p}^{\prime}(u-v)\left(y_{p}, t\right)\right)\right\} .
\end{aligned}\right.
$$

The study of the sign of each term of the sum in (6.2) shows with the entropy condition (1.4) (as in [6]) that, by conserving only the terms at $y_{p}=0$ and $y_{p}=\infty$, 
we have the inequality

$$
\frac{d}{d t} I(t) \leq \mu_{0}(t)-\mu_{\infty}(t)
$$

with $\mu_{0}(\cdot)$ and $\mu_{\infty}(\cdot)$ given by

$$
\mu_{a}(t)=\lim _{x \rightarrow a}\{\operatorname{sgn}(u(x, t)-v(x, t))(f(u(x, t))-f(v(x, t)))\} r(x), \quad t \geq 0,
$$

for $a=0+$ or $+\infty$.

To prove (3.5), it is sufficient to show that

$$
\mu_{0}(t) \leq\left|w_{0}(t)-z_{0}(t)\right|
$$

and

$$
-\mu_{\infty}(t) \leq 0 .
$$

Inequality (6.5) results from the fact that there do not exist incoming characteristic curves at $x=+\infty$, by Lemma 1 ; and hence, the values $u(\cdot, \cdot)$ and $v(\cdot, \cdot)$ are greater than $u_{*}$ (see (2.4)).

To establish the inequality $(6.4)$, we define $\gamma(\cdot)$ and $\delta(\cdot)$ by

$$
\gamma(t)=\lim _{x \rightarrow 0+} r(x) f(u(x, t)), \quad \delta(t)=\lim _{x \rightarrow 0+} r(x) f(v(x, t)), \quad t \geq 0 .
$$

For each positive number $t$, we distinguish between four cases:

(1) If $\gamma(t)=w_{0}(t)$ and $\delta(t)=z_{0}(t)$, it is obvious that $\mu_{0}(t)=\left|w_{0}(t)-z_{0}(t)\right|$.

(2) If $\gamma(t) \neq w_{0}(t)$ and $\delta(t)=z_{0}(t)$, then with the boundary condition (1.3.a) we have $\gamma(t) \geq w_{0}(t), \limsup _{x \rightarrow 0} a(u(x, t)) \leq 0$, and $\liminf _{x \rightarrow 0+} a(v(x, t)) \geq 0$. Thus we get

$$
\mu_{0}(t)=-\left(\lambda(t)-z_{0}(t)\right) \leq-\left(w_{0}(t)-z_{0}(t)\right) \leq\left|w_{0}(t)-z_{0}(t)\right| .
$$

(3) If $\gamma(t)=w_{0}(t)$ and $\delta(t) \neq z_{0}(t)$, we obtain (6.4) by the same arguments as in $(2)$.

(4) If $\gamma(t) \neq w_{0}(t)$ and $\delta(t) \neq z_{0}(t)$, then with (1.3.a) we have

$$
\limsup _{x \rightarrow 0+} a(u(x, t)) \leq 0 \text { and } \limsup _{x \rightarrow 0+} a(v(x, t)) \leq 0
$$

hence, $\mu(t)$ is nonpositive, because the function $f(\cdot)$ decreases between $u(x, t)$ and $v(x, t)$ for $x$ sufficiently small.

From (6.3)-(6.5) we deduce

$$
\frac{d}{d t} I(t) \leq\left|w_{0}(t)-z_{0}(t)\right|
$$

which yields inequality (3.5) by integration.

7. Extensions. We now view the different hypotheses (H.4)-(H.8) concerning the functions $f(\cdot)$ and $r(\cdot)$ (see $\S 2$ ). First, note that the points $x=0$ and $x=+\infty$ play a symmetric role: namely, instead of assumption (H.3) in Theorem 1 we can make the assumption (H.4), then we have to impose the boundary condition at the point $x=+\infty$. More precisely, the following result holds with a proof similar to the ones of Theorems 1-3. 
THEOREM 4. Under assumptions (H.1), (H.2) and (H.4), and if $r(\cdot) u_{0}(\cdot)$ is integrable and $r(\cdot) f\left(u_{0}\right)$ and $w_{0}(\cdot)$ bounded, there exists a solution $u=u(x, t)$ of problem (1.1), (1.2), (1.3.b) and (1.4). The uniqueness is specified by a semigroup property: for $u(\cdot, \cdot)$ and $v(\cdot, \cdot)$ piecewise continuously differentiable solutions associated with initial data $u_{0}(\cdot)$ and $v_{0}(\cdot)$ and boundary data $w_{\infty}(\cdot)$ and $z_{\infty}(\cdot)$, we have

$$
\begin{aligned}
\int_{0}^{\infty}\left|u\left(x, t_{2}\right)-v\left(x, t_{2}\right)\right| r(x) d x \leq & \int_{0}^{\infty}\left|u\left(x, t_{1}\right)-v\left(x, t_{1}\right)\right| r(x) d x \\
& +\int_{t_{1}}^{t_{2}}\left|w_{\infty}(s)-z_{\infty}(s)\right| d s
\end{aligned}
$$

for all $0 \leq t_{1} \leq t_{2}$.

The regularity and the explicit representation of the solution given by Theorem 4 are the same as the ones derived in Theorems 1 and 3.

Now consider the case where no boundary condition must be imposed.

THEOREM 5. Under Hypotheses (H.1), (H.2), (H.3.a) and (H.4.a) and if the function $r(\cdot) u_{0}(\cdot)$ is integrable and the function $r(\cdot) f\left(u_{0}(\cdot)\right)$ bounded, there exists a solution $u=u(x, t)$ of problem (1.1), (1.2) and (1.4), piecewise continuous, with

$$
\|r(\cdot) f(u(\cdot, t))\|_{\infty} \leq\left\|r(\cdot) f\left(u_{0}(\cdot)\right)\right\|_{\infty}, \quad t \geq 0 .
$$

If $u(\cdot, \cdot)$ and $v(\cdot, \cdot)$ are piecewise continuously differentiable solutions associated with initial data $u_{0}(\cdot)$ and $v_{0}(\cdot)$, we have

$$
\int_{0}^{\infty}\left|u\left(x, t_{2}\right)-v\left(x, t_{2}\right)\right| r(x) d x \leq \int_{0}^{\infty}\left|u\left(x, t_{1}\right)-v\left(x, t_{1}\right)\right| r(x) d x
$$

for $0 \leq t_{1} \leq t_{2}$.

Moreover, the function $u(\cdot, \cdot)$ is given explicitly by

$$
u(x, t)=f_{\varepsilon(x, t ; y)}^{-1}\left(\frac{c(x, t ; y)}{r(x)}\right), \quad x>0, t>0,
$$

where the point $y=y(x, t)$ minimizes the function $G(x, t ; \cdot)$.

Here, we take

$$
\begin{aligned}
G(x, t ; y)= & \int_{0}^{y} u_{0}(\xi) r(\xi) d \xi-t c(x, t ; y) \\
& +\int_{y}^{x} f_{\varepsilon(x, t ; y)}^{-1}\left(\frac{c(x, t ; y)}{r(\xi)}\right) r(\xi) d \xi
\end{aligned}
$$

with

$$
\varepsilon(x, t ; y)= \begin{cases}+1 & \text { if } 0<y<x \\ -1 & \text { if } x<y\end{cases}
$$

and

$$
t=\int_{y}^{x} h_{\varepsilon(x, t ; y)}(c(x, t ; y), \xi) d \xi
$$

Finally, we give the results in the case where we have to impose both boundary conditions at $x=0$ and $x=+\infty$, but for the sake of simplicity, we only view the cases where there is no free boundary problem both at $x=0$ and $x=+\infty$. 
THEOREM 6. Under Hypotheses (H.1), (H.2), (H.3.a) and (H.4.b) and if one of the following assumptions is satisfied:

(1) (H.5) and (H.8) hold,

(2) (H.6) and (H.7) hold,

(3) (H.6) and (H.8) hold, or

(4) (H.5) and (H.7) hold, with $w_{0} \equiv 0$ or $w_{\infty} \equiv 0$,

problem (1.1)-(1.4) is well posed: similar results as in Theorems 1-5 hold for this problem (existence, uniqueness and explicit representation).

For details, see $[\mathbf{1 0}, \mathbf{1 1}]$.

\section{REFERENCES}

1. C. Bardos, A. Y. Leroux and J. C. Nedelec, First order quasilinear erquations with boundary conditions, Comm. Partial Differential Equations 4 (1979), 1017-1034.

2. S. N. Kruskov, First order quasilinear systems in several independant variables, Math. USSR Sb. 10 (1970), 217-243.

3. P. D. Lax, Hyperbolic systems of conservation laws, Comm. Pure Appl. Math. 10 (1957), 537-566.

4. P. D. Lax, Hyperbolic systems of conservation laws and the mathematical theory of shock waves, SIAM, Philadelphia, Pa., 1973.

5. O. A. Oleinik, Discontinuous solutions of nonlinear differential equations, Amer. Math. Soc. Transl. (2) 26 (1963), 95-172.

6. B. L. Keyfitz, Solutions with shocks, an example of $L^{1}$ contractive semi-groups, Comm. Pure Appl. Math. 24 (1971), 125-132.

7. M. E. Schonbek, Existence of solutions to singular conservation laws, SIAM J. Math. Anal. 15 (1984).

8. J. A. Smoller, Reaction-diffusion equations and shock waves, vol. 258, Springer-Verlag, 1983.

9. G. B. Whitham, Linear and nonlinear waves, Wiley-Interscience, New York, 1974.

10. Ph. Le Floch and J. C. Nedelec, Explicit formula for weighted scalar conservation laws, Centre Math. Appl. Ecole Polytechnique, preprint, January 1985.

11. Ph. Le Floch, Contributions à l'étude théorique et à l'approximation numérique des systèmes hyperboliques nonlinéaires, Thèse, École Polytéchnique, France.

Ecole Polytechnique, Centre de Mathématiques Appliquées, 91128 PalaiSEAU CEDEX, FRANCE 